\title{
Coherence, Consistency, and Cohesion: Clade Selection in Okasha and Beyond ${ }^{1}$
}

\author{
Matt Haber \\ Department of Philosophy and Center for Population Biology, UC Davis \\ Andrew Hamilton \\ Department of Philosophy and Science Studies Program, UC San Diego
}

\begin{abstract}
Samir Okasha argues that clade selection is an incoherent concept, because the relation that constitutes clades is such that it renders parent-offspring (reproduction) relations between clades impossible. He reasons that since clades cannot reproduce, it is not coherent to speak of natural selection operating at the clade level. We argue, however, that when species-level lineages and clade-level lineages are treated consistently according to standard cladist commitments, clade reproduction is indeed possible and clade selection is coherent if certain conditions obtain. Despite clade selection's logical coherence, however, we share some of Okasha's pessimism. Whether or not clades are a unit of selection is ultimately a question of empirical support and theoretical import, but we offer reasons to be skeptical about clade selection as a research programme.
\end{abstract}

\section{Introduction}

In a recent article, Samir Okasha argues that selection for clades-described as groups of species that include a common ancestor and all its descendents-is either species selection by another name, or is conceptually incoherent because clades do not reproduce (Okasha 2003, 749). We argue, however, that in the absence of good reasons to do otherwise, consistency demands that standard cladist concepts of species, species extinction, and the species parent-offspring relation-commitments Okasha acceptsshould be used in the generation of cladist analogues of these concepts as applied to clades. In Section 3 we show how such concepts can be understood by offering an analysis of a species-level lineage and the clade-level relationships that arise from it. On our view, clade selection is both distinct from species selection and is conceptually coherent for the cladist if species selection is.

Mere coherency, however, is too a low a threshold by which to judge the quality of a concept. In Section 4 we ask whether the cladist view of clade selection is empirically plausible or of theoretical import. Here we are more pessimistic, and argue that (i) competition among clades is precluded in ways that it is not precluded among species, (ii) clades lack the relevant cohesion generating relations (CGRs) (and subsequent disruptions) that are present at the level of species, and (iii) clades seem only rarely to share selective environments. ${ }^{2}$ It is these differences that ultimately allow species- and clade-level lineages to be treated differently by the cladist with respect to a functional

\footnotetext{
${ }^{1}$ Thanks are due both to Jim Griesemer and to the Philosophical Pizza Munchers for helpful discussions of the issues under consideration here. This work is fully collaborative: the authors are listed alphabetically. ${ }^{2}$ Elihu Gerson first suggested this point about CGRs (and what to call them) to us. He should not be held responsible, however, for what we've done with it.
} 
account of selection $^{3}$ (though not with respect to such basic cladist concepts as the parentoffspring relation or the generation-extinction relation). Consistency does not require cladists to be committed to clade selection merely because it is coherent.

\section{Okasha On Clade Reproduction}

Okasha's central claim is that the concept of clade selection is incoherent because clades do not reproduce. The motivation is easy to see. Every responsible account of how selection works has it that selection modifies the composition of a population-be it cells, genes, organisms, demes, species, or clades - through the differential reproduction of heritable traits (Lewontin 1970; Maynard Smith 1987). If clades don't reproduce, however, it is incoherent to claim that they are a locus of selection.

According to Okasha, clades cannot be said to reproduce because clades do not meet two necessary conditions for reproduction. Namely, the conditions that like must beget like (LMBL) and that offspring must be capable of independent existence (IE). These conditions can be stated as follows:

(LMBL) Parent and offspring must both be entities at the same level in the biological hierarchy. (Okasha 2003, 743)

\begin{abstract}
"An act of reproduction must result in the production of an offspring entity which has an independent existence of its parental entity, in the sense that it can continue to exist when its parent dies, at least in principle. [...] ...[I]f two entities are related as parent and offspring, it must be possible for them to become related as ancestor and descendant in the future.” (Okasha 2003, 743)
\end{abstract}

According to Okasha, clades fail to meet both the LMBL and IE conditions-and thus do not reproduce-because a clade just is a monophyletic group of species, where monophyly is understood to mean that clades are comprised of an ancestral species and all of its descendents (Okasha 2003,745; Hennig 1966; Wiley 1981). This being the case, Okasha reasons, every relation between a more inclusive clade and a less inclusive one is by definition a relation of a whole to a proper part, rather than a parent-offspring relation. The simple cladogram in Figure 1 illustrates Okasha's thinking.

\footnotetext{
${ }^{3}$ This presumes, of course, that there is a meaningful distinction between species and clades. This is not, however, a view held by all cladists; some contend that the rank of species is unhelpful and ought to be abandoned (Mishler 1999). We can ignore this, however, as Okasha's position could still be articulated, and, we believe, our response would still stand.
} 


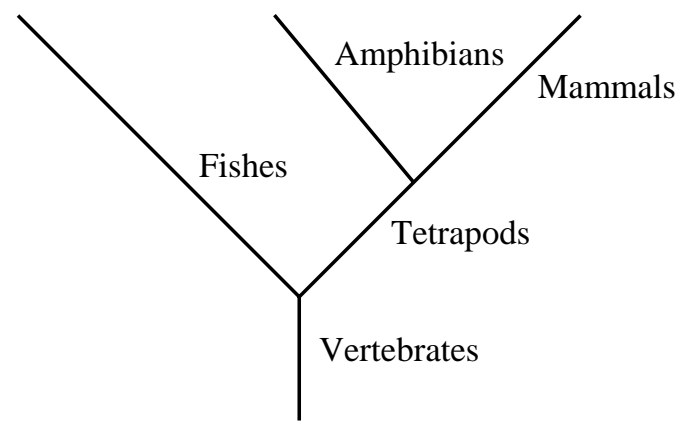

Figure 1. Simple cladogram showing lineage relations among vertebrates. More inclusive clades are at the bottom, with clades (sub-clades) becoming increasingly less inclusive toward the top.

On Okasha's reading of cladistics, the clade 'tetrapods' is contained as a proper part within the clade 'vertebrates', just as the clades 'mammals' and 'amphibians' are contained as proper parts within the clade 'tetrapods'. These clade relations do not satisfy the necessary conditions for reproduction relations because:

1. The ancestor of any of the less inclusive clades toward the top of the cladogram cannot be a clade; rather, "it can only be a species" (Okasha 2003, 745). Thus, the LMBL condition is violated.

2. The clade 'tetrapods' cannot outlive the clade 'vertebrates'. If the vertebrates go extinct, all the tetrapods will go extinct as well, since "the only way a monophyletic clade can cease to exist is if all its constituent species go extinct, which implies that all the sub-clades which are parts of it must cease to exist too" (Okasha 2003, 745). Thus, the IE condition is violated.

These two claims mirror Okasha's general thesis about clade selection. The first, if true, will show that, ceteris paribus, clade selection is species selection by another name, since the relevant ancestor is a species rather than a clade. In this case we have a species begetting the descendent species that make up clades, not clades begetting clades. The second claim, if true, will show that clade selection is incoherent because the relations between clades that monophyly requires will not allow less inclusive clades to exist independently of more inclusive ones.

We accept LMBL and IE as necessary conditions for reproduction and grant that if either (1) or (2) prove to be the case, there fails to be a reproduction relation and clade selection either collapses into species selection or becomes conceptually incoherent. In the next section, however, we show that cladists are not forced to accept (1) or (2), since they can apply their analysis of species concepts to clades in just the way that consistency demands they should, as well as in a way that respects the LMBL and IE conditions.

\section{The Cladist Concept of Species and Its Clade-Level Analogue}

Okasha's argument presumes a cladist perspective. Here, we accept this perspective without debate, and our arguments should be understood as operating within the accepted canons of cladism. Broadly speaking, we take the cladist perspective to be a commitment 
to representing ancestral conditions and evolutionary relationships using phylogenetic systematics as a framework. More specifically to the case at hand, cladists are committed to the unintuitive but useful notion that "when a lineage splits the ancestral species automatically goes extinct, even if it is phenotypically indistinguishable from one of the daughter species" (Okasha 2003, 740). That is, whenever there is a speciation event, two new species are created, even if one of the new species has all the properties of the ancestor species - indeed, even if one of the new species is populated by all the same individuals as the ancestor species. Figure 2 graphically illustrates this view of lineage splits (though care needs be taken to not confuse cladograms with phylogenetic trees; Sober 1991). Notice that Species $a$ goes extinct at the speciation event that occurs at time $t_{1}$, even if most or all of the individuals (or their phenotypically unmodified progeny) constituting Species $a$ persist until time $t_{2}$. As Okasha points out, this convention is neutral with regard to what constitutes the speciation event (Okasha 2003, n. 8).

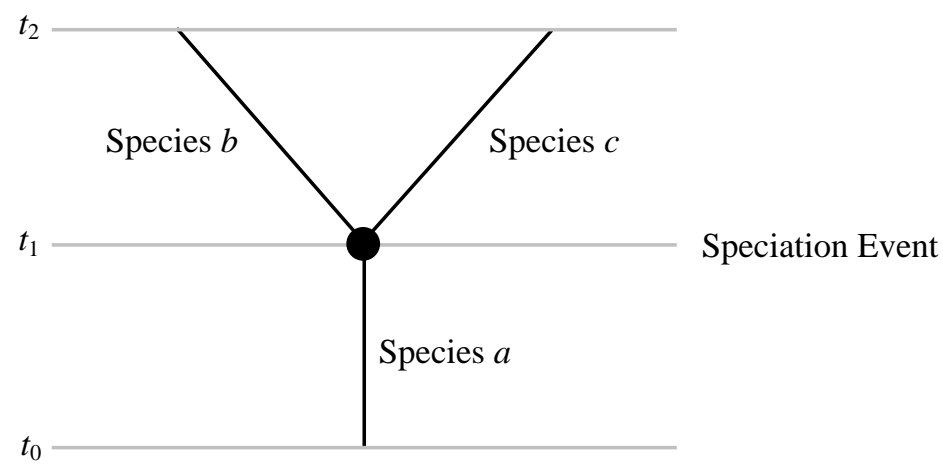

Figure 2. Diagram of cladist species relationships before and after speciation.

We will accept without argument Okasha's account of the conventional cladist treatment of lineage splits. In fact, this treatment constitutes the core of our response. We simply ask what happens when we require a consistent treatment of lineage splits across levels of lineage. ${ }^{4}$ After all, if the question to be decided is whether clade selection is coherent, then we ought to be looking at lineages of clades-not, as Okasha does, at lineages of species. When we do, we see that monophyly is not fatal to the parent-offspring relation for clades (of which much more below), and that statement (2) above is ambiguous; disambiguation here results in conflicting truth values. To see this, consider that for the cladist, extinctions do not occur only when all the members of a given group cease to exist; by definition, generation events are also extinction events whether any of the members of the progenitor group die or not.

In light of the two senses of 'extinction' it will be useful to introduce a distinction between them. Consider two ways a cladist might deem a species to have gone extinct. There is first the sense in which Okasha uses the term, namely that all the parts of a species may cease to exist (i.e., all the individual organisms of a species die). Second there is the sense in which a speciation event entails the extinction of a species-despite

\footnotetext{
${ }^{4}$ Okasha adopts a view on which levels are differentiated according to the standard biological hierarchy that runs from cells to organisms to species and so on. We accept this view, noting as Okasha does, that there is much more to say.
} 
the survival of some (or, indeed, all) of the individual organisms of that species. Let's call the former kind of extinction Type I extinction, and the latter Type II extinction. To those familiar with evolutionary theory, neither type of extinction should come across as unintuitive. Beyond the realm of professional biologists, though, Type II extinction is far less intuitive. To convince yourself of this, consider why it might be difficult to garner general sympathy for a looming Type II extinction of a charismatic megafauna. 'Save the Whales' has much less political expediency if whale populations are booming despite the looming reproductive isolation of a particular population of whales!

What we are urging is that without good reasons to do otherwise, consistency will require cladists to apply both senses of extinction to clades as well as species. Type II extinctions are applied to species, in part, to allow for the generation of clean, bifurcating lineages, i.e., it is a useful model for representing phylogeny. A similar justification can be appealed to for applying type II extinctions to clades, allowing clade lineages to be modeled. E.g., applying type II extinctions to species eliminates the difficulty of dealing with anagenesis in phylogenetic reconstruction; applying type II extinctions to clades eliminates analogous anagenetic change of compositions of clades. To see the force of this re-orientation of Okasha's discussion toward clade-level lineages, consider Figure 3, in which the monophyletic lineage of a single species, species $a$, is shown as it persists through five time slices. It should be noted that $a-i$ are all species. The relations in the species lineage cladogram on the left side of Figure 3 are those required by the cladist conception of species: each speciation event results in two new species and the extinction of the immediate ancestor species. Both of Okasha's conditions are met in this case. Every lineage split has species begetting species, satisfying the LMBL condition. Also, younger species can outlive their parent species, satisfying the IE condition. It is worth noting that the satisfaction of the IE condition is entailed by the cladist treatment of lineage splits. By definition, the parent species goes extinct at every speciation event. Again, this holds true even if there is a persistence of all the parts of a species across the speciation event (i.e., the individual organisms comprising the parent offspring survive across $\left.t_{1}\right)$. 
Species Lineage

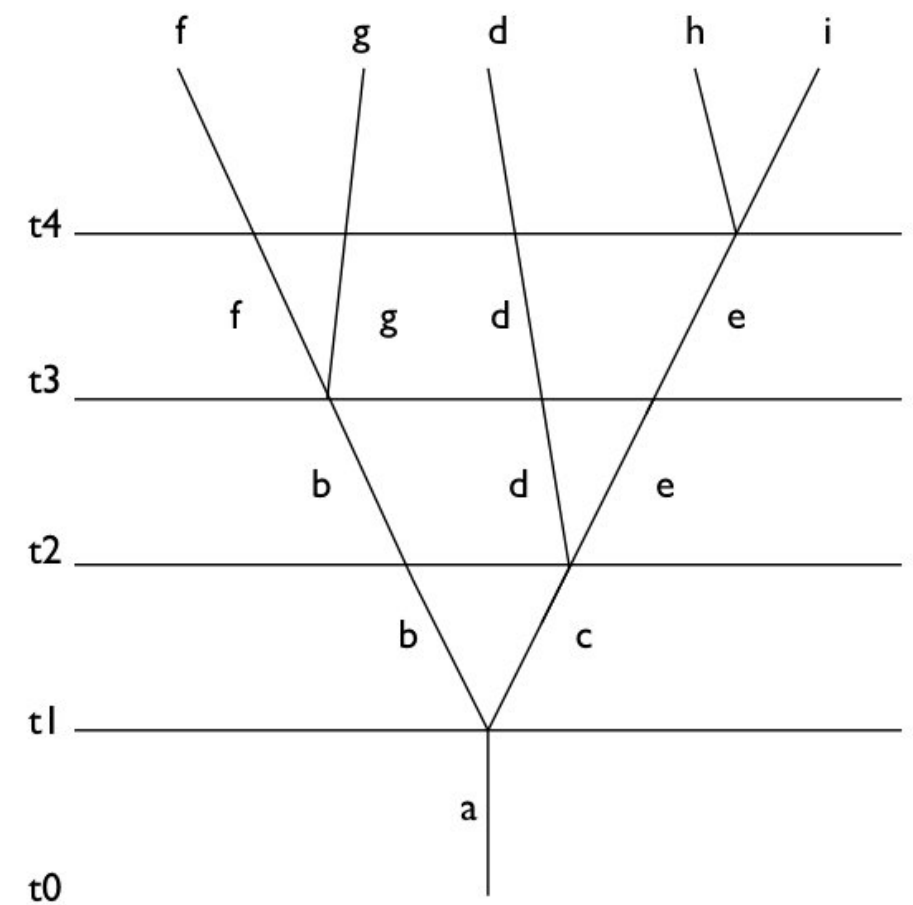

Clades

Generated Extinct Persisting

$$
8=\text { e:h,i } \quad 4=\text { a:f,e } \quad 5=\text { b.f,g }
$$

$7=\mathrm{c}: \mathrm{d}, \mathrm{i}$

$6=\mathrm{a} \cdot \mathrm{f}, \mathrm{i}$

\section{Generated Extinct Persisting}

$5=\mathrm{b}: \mathrm{f}, \mathrm{g} \quad 2=\mathrm{a}: \mathrm{b}, \mathrm{e} \quad 3=\mathrm{c}: \mathrm{d}, \mathrm{e}$

$4=\mathrm{a} \cdot \mathrm{f}, \mathrm{e}$

Generated Extinct

$3=c: d, e \quad I=a: b, c$

$2=a: b, e$

Generated*

$\mathrm{I}=\mathrm{a}: \mathrm{b}, \mathrm{c}$

Figure 3. Species-level cladogram with its progression of clade lineages displayed on the right. Clades are numbered, and have been individuated by listing the ancestor species before the colon and the two most widely separated terminal species after the colon. Some cladists might include a clade ' 0 ' at $t_{0}$ consisting solely of species lineage ' $a$ '. We note this preference by marking Clade 1 with an asterisk.

What worries Okasha about applying this analysis to clades is the cladist definition of clades as monophyletic groups of species. He takes it that this definition requires us to construe the relations between the younger clades and the older clades of a particular lineage as a logical relation of proper parts to a whole, such that if a more inclusive clade ceases to exist, all less inclusive clades that are its parts would have to cease to exist as well:

....[C]lades are by definition monophyletic, and as a matter of logic, monophyletic clades cannot stand in ancestor-descendent relations with one another: if all higher taxa are required to be monophyletic, then ancestral higher taxa do not exist. A taxon which contains all the descendants of its members as proper parts cannot be ancestral to any other such taxon. (Okasha, 745)

The challenge presented here is how to retain the part-whole relationship inherent in monophyly while at the same time honoring the motivation behind the cladist requirement that lineage splits always result in the generation and extinction of taxa. Lacking some principled distinction between lineage splits at one level and lineage splits 
at another, this requirement can apply to clades as well as species: on this view, older clades must go extinct if there are to be any newer clades at all.

If consistency is to be any guide, the cladist's monophyly requirement cannot mean that we should always understand younger clades and older clades to stand in part-whole relations. How, then, should we understand relationships between monophyletic older and younger clades? The answer to this question is suggested by the progression of clades displayed on the right-hand side of Figure 3. Notice that at any given time slice, clades persist, go extinct, or are generated from those that go extinct, in just the same way as the species lineage is analyzed in the cladogram on the left. At $t_{2}$, for instance, there is an extinction and bifurcation: Clade 1 goes extinct because of a cladogenic event that gives rise to Clades 2 and 3 .

We, along with Okasha, recognize that clades, like species, are concrete spatiotemporal entities, rather than atemporal classes or sets (Ghiselin, 1974, 2002; Hull 1976, 1980). The principal difference between our analysis and Okasha's is that we treat clades synchronously, whereas Okasha treats clades diachronically. To treat clades synchronously is just to recognize that phylogenetic relations obtain at a time. Clades may exist diachronically, but the 'sister-group' relation is synchronous. This is similar to the 'sibling' relation; the sibling relation obtains at a time between two brothers, even if that relation depends upon historical facts and persists over time. Treating clades synchronously recognizes a vital distinction between cladograms and phylogenetic trees; phylogenetic trees display historical information, cladograms display sister-group relationships that obtain between lineages. (See Mishler and Theriot (2000) for a fuller account of synchronic treatment of clades, and for a connection to Hennig's treatment of the tokogenetic/phylogenetic relationship distinction.) This allows us to introduce a time index that treats clades as concrete spatiotemporal entities that exist and cease to exist at particular times. This is entirely consistent with the cladist treatment of species, which are not thought of as abstract classes, but as real spatiotemporal entities that engage in lineage-relevant behaviors. As the cladogram in Figure 3 shows, species can be tracked as they reproduce, succeed, fail, go extinct, and so on. We suggest that analogous events can be tracked of clades when they are treated synchronously. We grant that clades are scattered entities, on our analysis, but this should not be considered an obstacle to being an individual in the same way that species are individuals (Baum 1998; Ereshefsky 2001; Ghiselin 1997).

Okasha's mistake is that he fails to move from the consideration of cladograms displaying species-level lineages to something analogous displaying clade-level lineages. The mistake is akin to looking at population level lineages of a single species and trying to infer coherency conditions of species selection. This is understandable, as clade-level cladograms are not widely available (for the simple reason that biologists do not, at present, produce clade level-cladograms). Furthermore, drawing clade-level lineages turns out to be a very difficult task. ${ }^{5}$ In the end, whether clade-level lineages are easy or

\footnotetext{
${ }^{5}$ Ultimately, we will leave this job to cladistic theorists - if they should so choose to take on such a task. We did conceive of a few ways to display clade lineages. Some of these resembled species lineages, though we had to allow for non-bifurcating parent-offspring relationships. Other clade-level lineages took on different forms than the traditional species-level cladogram. Ultimately, these complexities leave us unworried. After all, there is no reason to expect that representations of lineages at different levels need conform to something resembling a species-level cladogram (i.e., this is not a necessary condition from the
} 
difficult to draw or conceive of is a separate issue from that of treating clades as individuals.

Moreover, a synchronic treatment of clades explains why monophyly should not be considered a fatal obstacle to clade reproduction. The monophyly condition is typically regarded as satisfied when an ancestral species and all its descendants are the parts composing a clade. But monophyly cannot be understood this way if we treat clades synchronously. The reason for this is straightforward: at any given time slice, a clade may not consist of an ancestral species and all its descendants for the simple reason that the ancestral species may no longer exist at that time slice. Consider again Figure 3. At $t_{3}$ both species $b$ and species $c$ are extinct, so no clade at $t_{3}$ may include either $b$ or $c$ as proper parts.

How, then, should we understand the monophyly requirement while construing clades synchronously? The answer, we propose, is that for an individual clade to be monophyletic is simply for the parts of that clade, at any given time slice, to stand in the proper inclusivity relationships. That is, that the parts of a clade should consist of all and only the extant descendants of some ancestral species. Furthermore, as suggested above, it cannot be the case that monophyly requires that an ancestral species (or any extinct species) be a part of the clade it specifies. Extinct ancestral species, though, may be a part of an extinct ancestral clade in the clade lineage of any given clade. By failing to consider clades synchronously from a cladist perspective, Okasha conflates the notion of older and younger clades with the broad whole-part relations that he takes monophyly to require. We argue, however, that monophyly relationships can be retained at any given time slice while respecting the IE condition for relationships among clades. On this analysis, each clade identified in Figure 3 is monophyletic: each contains all the extant offspring of some ancestral species. Notice also that the clades in Figure 3 may be inclusive of each other as proper parts. At $t_{4}$, for example, Clade 6 includes Clade 7 , which in turn includes Clade 8. Thus, the appropriate inclusivity requirements of monophyly are retained among clades precisely because at any given time slice the appropriate part-whole relations hold.

The clade relationships in Figure 3 satisfy the LMBL condition as well, since it is clearly not the case, as Okasha alleges, that "if we ask what the ancestor of [a given clade] is, then the answer can only be a species, not another clade” (Okasha 2003, 745; emphasis in the original). While it is true that each cladogenic event is precipitated by a speciation event, it is not simply the case in the present example that species are begetting clades. To see this, consider any of the younger clades. Clade 7, for instance, is generated at $t_{4}$, but its ancestor species, species c, has been extinct since $t_{2}$. Species $c$, then, cannot be said to be the parent of the clade. Clade 7 arises from a species-level lineage splitspecies $e$ goes extinct giving rise to species $h$ and $i$ at $t_{4}$ - but species $e$ cannot be the parent of the clade, because the clade includes species $d$, species $e$ 's sister species (both of them having arisen at exactly the same time as the result of the same speciation event at $t_{2}$ ). What does make sense is to say that Clade 7 was begotten by Clade 3 . What we are urging, after all, is that Clade 3 can be understood as an entity that was generated from a Type II extinction event at $t_{2}$, persisted through $t_{3}$, and went Type II extinct at $t_{4}$ precisely

cladist perspective). Consider how a cladist might represent the lineage of a unicellular organism, or of a population of sexually reproducing organisms, or of a familial lineage. 
because Clade 7 came into existence when species $e$ went extinct and gave rise to species $h$ and $i$.

Furthermore, care must be taken to distinguish what counts as the 'parent' of an entity and what counts as the 'ancestor' of an entity. We do not deny that clades do indeed have species as ancestors. This, though, does not preclude that clades also have clades as ancestors (and parents). To see why this is so, consider the species lineage in Figure 3. Every lineage split has species begetting species. This description, though, is in part a function of the level of description of the lineage. If we were to describe the speciation events at a more fine-grain level, then we might describe species as having particular populations (or demes?) as their ancestors, yet this fact does not preclude us from meaningfully describing species as begetting species. This analogy may be clearer if we think about humans. Any human is both the descendant of a single cell and of other humans; identifying both as ancestors presents no conflict. It is simply not the case that a single level has exclusive status as an ancestor. The same cannot be said of the parentoffspring relation. Okasha is correct that for two entities to stand in a parent-offspring relation they must be of the same kind. However, as discussed above, the parentoffspring relation can be rendered coherent for clades. ${ }^{6}$

It is worth reiterating that translating the progression of clades from Figure 3 into a clade-level cladogram may prove difficult. Complexity, though, ought not be confused with incoherency. Furthermore, conceptualizing of clades and clade reproduction/extinction as suggested above may strike some as unintuitive. ${ }^{7}$ However, this ought not be surprising when we consider some of the unintuitive properties a cladist perspective of species and species reproduction/extinction entails.

\section{Clade Selection: Beyond Mere Coherency?}

That a coherent case can be made for clade reproduction has important consequences for Okasha's argument. Okasha rejects Sterelny and Vermeij's claim that clades possess adaptations-i.e., "heritable characters" — on the grounds that clades are not the kinds of things that can reproduce (Okasha, 744). But this turns out to be insufficient grounds on which to base such a rejection. Whether clades are the kinds of things that are capable of possessing adaptations, or competing for resources, or are in actuality a locus of selection remains an open question. Here we go beyond considering mere coherency, and ask whether one should be optimistic about the results of a clade selection research programme.

Several reasons for pessimism suggest themselves. First, if competition at a particular level is a necessary condition for the operation of natural selection at that level, clade selection can be shown to have a problem-somewhat akin to the one Okasha tries to motivate for coherency - that species selection does not have. The problem is that some clades share parts with other, closely related clades, rendering talk of competition between those clades incoherent. In Figure 4, for example, clades 1 and 2 share extant

\footnotetext{
${ }^{6}$ Alternatively, the parent-offspring relation may be rendered irrelevant to the production of lineages for things like clades. The stance taken towards this problem is likely to be similar to the stance taken towards the question of whether a parent-offspring relation exists between an asexual unicellular organism and the resultant daughter cells that occur after division (i.e., does the original organism persist?) For now, we will leave unanswered the question of what kinds of processes are sufficient to create lineages; suffice to say that reproduction qualifies as a lineage generating process.

${ }^{7}$ Indeed, we ourselves find it quite unintuitive!
} 
members at $t_{\mathrm{n}}$. These clades overlap-Clade 1 includes extant members of Clade 2-in such a way that it will not make sense to speak of competition between them, though it will make sense to speak of competition between the extant species that constitute them.

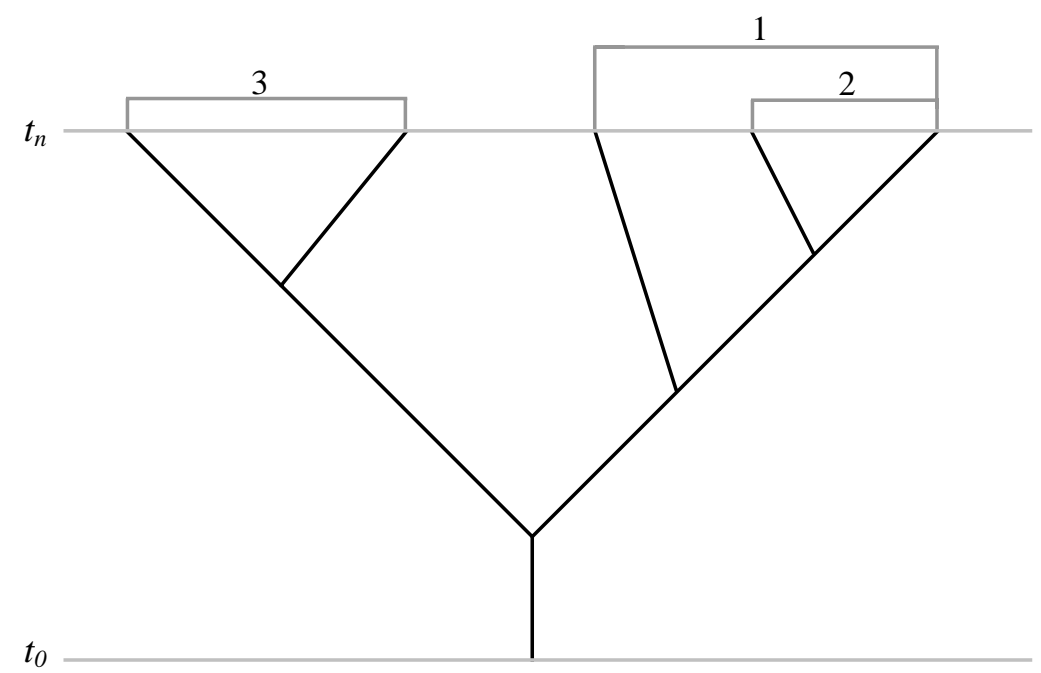

Figure 4: Simple cladogram showing clades with overlapping parts.

This suggests a third condition necessary for clade selection, on the assumption that competition is necessary for selection to occur at a given level:

(NOP) Competing clades must have no overlapping parts.

We suspect that part of what led Okasha to incorrectly conclude that clade selection is incoherent is that his analysis included clades that failed to satisfy the NOP condition (see Okasha, 2003, fig. 2). This condition is not fatal to clade selection, as the cladogram in Figure 4 shows. Clade 3 does not share extant parts with Clade 1 or Clade 2, and it is therefore not incoherent to speak of competition between Clade 3 and the other clades. Whether or not competition occurs between clades that meet the NOP condition is a matter for empirical investigation. Our hope is that by formulating NOP as a necessary condition for competition among clades, we will help investigators identify which clades to include and exclude as possible candidates for competition.

Second, and perhaps more importantly, we ask whether the relationships in which cladists are interested at the species level-those that are empirically or theoretically important-also obtain at the clade level. One way to explore this question is to consider Type II extinctions. Why, according to the cladist, do parent species go extinct at a speciation event? Though the specific concept of speciation is irrelevant, what matters is that some speciation event occurred. The answer seems to be that the cohesion generating relationships (CGRs) among the parts of a species change in a relevant manner such that new species are generated. The cladist marks (Salmon, 1994; Griesemer (in review)) this as an extinction of the parent species along with the generation of two daughter species as a way of representing the disruption of one or more 
of these CGRs. That is to say that the magnitude of disrupting a CGR is considered great enough to constitute an extinction event.

To illustrate this point, consider how a cladist might treat speciation using the Biological Species Concept (BSC) (Mayr 1942). From this point of view a candidate CGR is that all the parts of a species can, in principle, successfully reproduce with one another. A speciation event constitutes the disruption of this CGR among the parts of a species, and is marked as such by the cladist with an extinction and generation event.

What is at stake here is whether there are analogous CGR disruptions at the level of clades, and whether marking such disruptions is suitably interesting. This is not a logical coherency question, but a question of theoretical import and empirical evidence. But this is not our question to answer. Rather, the onus falls on clade selection advocates to identify relevant CGR disruptions. Ultimately, we recognize that to those evaluating clade selection, clade level CGR disruptions may prove to be uninteresting. As it stands, we share Okasha's pessimism regarding clade selection. It is the lack of good evidence of appropriate CGRs among the parts of clades that make clades poor candidates for selection. What we have provided, though, is both a mechanism to justify treating clades and species differently with regard to selection-species have the relevant CGRs while clades may not - and a research question for those working within a clade selection research programme.

A third reason for pessimism is that even supposing that clades could be identified as possessing an appropriate CGR, other conditions for selection seem unlikely to be satisfied. Notably, individual clades rarely (if ever) share a selective environment, leaving it unclear how something like heritability might apply to clades. Again, the question of shared selective environment is not one of coherency, but instead is an empirical question. It strikes us that clades are unlikely to satisfy this condition. The best bet is something resembling avatar selection.

Since whole species are rarely confined to a single local community, it will be convenient in the following discussion to introduce a term referring to the population of a species found in a particular community. I will call such a population an avatar of the species. It is the local 'embodiment' or 'representation' of the species in the local community. (Damuth 1985, 1137)

We suggest that the avatar concept can be extended to clades. A clade avatar would be the 'population' of a clade found in a particular community; the embodiment of a clade in a local community would be the presence of species of that clade in that community. Clade avatars share a selective environment by virtue of occupying a common locality. Furthermore, a community may consist of several different clade avatars.

Clade selection, then, would simply be a special case of clade avatar selection, where an entire clade consisted of a single clade avatar. Though this condition may be unlikely, it is certainly possible. Candidate cases in which clade avatar selection collapses into clade selection would be those in which the selective environment spans a geographic area containing all the members of a clade or cases where the selective environment is planet-wide (e.g., K-T boundary extinction events). 


\section{Conclusion}

Clade selection presents many conceptual difficulties. Not the least of these is that the cladists' unintuitive notion of Type II extinctions becomes even less natural when it is applied — as consistency demands it must — to clades. However thorny clade selection might prove to be, though, it has not been shown to be incoherent by Okasha's arguments. In spite of this, we think Okasha is not terribly far off the mark, since there are good empirical and theoretical reasons to be pessimistic about whatever fruits there may be of the cladist research programme.

All hope is not lost for advocates of clade selection, however, since the analysis here suggests some paths for future research. We believe that identifying the NOP condition as a necessary requirement for competition among clades constitutes a significant contribution to the field, as it will focus empirical work on those cases in which positive results are at least possible. We have also described other conditions necessary for clade selection (e.g., shared selective environment), and believe that together these constitute parts of a research programme for advocates of clade selection - though perhaps not a very appealing one.

Clade selection holds disquieting problems for the cladist, even if coherency is not one of them. It is not worries about coherency that should keep the cladist awake at night; rather, the cladist should be concerned about whether the theoretical and empirical worries surrounding clade selection can be met when coherency is assured. 


\section{References}

Baum, David (1998), “Individuality and the Existence of Species Through Time,” Systematic Biology, 47: 641-653.

Damuth, John (1985), “Selection Among 'Species': A Formulation in Terms of Natural Functional Units,” Evolution, 39(5): 1132-1146.

Ereshefsky, Mark (2001), The Poverty of the Linnaean Hierarchy: A Philosophical Study of Biological Taxonomy, Cambridge: Cambridge University Press.

Ghiselin, Michael (1969), The Triumph of the Darwinian Method, Berkeley: University of California Press.

_ (1974), “A Radical Solution to the Species Problem,” Systematic Zoology 23: 536544.

— (1997), Metaphysics and the Origin of Species. Albany, NY: SUNY Press.

— (2003), “Species Concepts: The Basis for Controversy and Reconciliation,” Fish and Fisheries, 3: 151-160.

Griesemer, James (In Review) “Tracking Organic Processes: Representations and Research Styles in Classical Embryology and Genetics.”

Hennig, Willi (1966), Phylogenetic Systematics. Urbana, IL: University of Illinois Press. Hull, David (1976), “Are Species Really Individuals?” Systematic Zoology 25:174-191.

— (1980). “Individuality and Selection,” Annual Review of Ecology and Systematics, 11: 311-332.

Lewontin, Richard (1970), “The Units of Selection,” Annual Review of Ecology and Systematics, 1: 1-18.

Maynard Smith, John (1987), “How to Model Evolution,” In John Dupre, (ed.), The Latest on the Best: Essays on Evolution and Optimality, Cambridge MA: MIT Press.

Mayr, Ernst (1942), Systematics and the Origin of Species, New York: Columbia University Press.

Mishler, Brent D. (1999), “Getting Rid of Species,” In R. Wilson (ed.), Species: New Interdisciplinary Essays, Cambridge, Mass: MIT Press. 307-315. 
Mishler, Brent D. and Edward C. Theriot (2000), “The Phylogenetic Species Concept (sensu Mishler and Theriot): Monophyly, Apomorphy, and Phylogenetic Species Concepts," In Quentin D. Wheeler and Rudolf Meier, (eds.), Species Concepts and Phylogenetic Theory, New York: Columbia University Press. 44-54; 119-132; 179-184.

Okasha, Samir (2003), “Does the Concept of “Clade Selection” Make Sense?,” Philosophy of Science, 70 (4): 739-751.

Salmon, Wesley, (1994), “Causality without counterfactuals,” Philosophy of Science 61: 297-312.

Sober, Elliot. (1991), Reconstructing the Past: Parsimony, Evolution, and Influence. Cambridge, MA.: MIT Press.

Wiley, E.O. (1981), Phylogenetics: The Theory and Practice of Phylogenetic Systematics, New York: Wiley-Interscience. 